\title{
Oral tolerance, an active immunologic process mediated by multiple mechanisms
}

\author{
Howard L. Weiner
}

See related article,

pages 1031-1038.

Center for Neurologic Diseases, Brigham and Women's Hospital and Harvard Medical School, Boston, Massachusetts, USA

Address correspondence to: Howard L. Weiner, Brigham and Women's Hospital, Center for Neurologic Diseases, 77 Avenue Louis Pasteur, HIM 730, Boston, Massachusetts 02115-5817, USA. Phone: (617) 525-5300; Fax: (617) 525-5252; E-mail: Weiner@cnd.bwh.harvard.edu.

Oral tolerance, the specific suppression of cellular and/or humoral immune responses to an antigen by prior administration of the antigen by the oral route, probably evolved to prevent hypersensitivity reactions to food proteins and bacterial antigens present in the mucosal flora. As with other forms of immunologic tolerance, oral tolerance also provides mechanisms to suppress pathologic reactivity against self and, thus, to prevent or treat autoimmune diseases.

\section{Tolerance as an active process}

Three assumptions were implicit in the classical concept of tolerance, as the term was originally used by Burnet (1). First, the primary function of the immune system was seen as defense of the organism against pathogens or, in a broader sense, against non-self materials. Second, in order to perform this function, the primary type of immunologic response was thought to be inflammation. Third, since the operation of the immune system is driven by its reactions to foreign pathogens, tolerance was envisioned as the simple absence of an immune response, accomplished by neonatal deletion of "forbidden" B- or T-cell clones, which recognize self epitopes.

With a better understanding of the immune system, it is now clear that tolerance is a much more complicated and diverse process. Autoreactive immune cells, such as those reacting against brain antigens, thyroglobulin, serum albumin, collagen, and other autoantigens, are, in fact, not deleted but are present in all individuals $(2,3)$. These cells not only remain harmless under normal conditions but may have an important function in maintaining tissue homeostasis and may be differentially expanded or focused depending on the tissue and the autoantigen (4). Furthermore, immunologic tolerance does not appear to rest on a sim- ple discrimination between self and non-self. Rather, the immune system reacts efficiently to a variety of molecular danger signals and often tolerates antigens that occur in the absence of such signals (5). Thus, immunologic tolerance cannot rely solely on neonatal deletional events, but must involve an active process that functions during the entire life of the organism.

A more appropriate concept of tolerance would include any mechanism by which a potentially injurious immune response is prevented, suppressed, or shifted to a noninjurious class of immune response. Tolerance is related to productive self-recognition rather than blindness of the immune system to its autocomponents. Mechanisms by which immune tolerance can be achieved, and their relationship to oral tolerance, are listed in Table 1.

Oral tolerance, in this broader sense, is of unique immunologic importance, since it is a continuous natural immunologic event driven by exogenous antigen. Due to their privileged access to the internal milieu, antigens that continuously contact the mucosa represent a frontier between foreign and self components. Oral tolerance evolved to treat external agents that gain access to the body via a natural route as internal components without danger signals, which then become part of self. Hence, it would seem logical that autoimmune diseases caused by an inappropriate response to self antigens might ultimately be treated by presenting such autoantigens to the mucosal surface, where they can be dealt with in a noninjurious (noninflammatory) immunologic environment (6).

\section{Dose effects and oral tolerance \\ Like tolerance itself, oral tolerance is an active immunologic process that is mediated by more than one mecha- nism and is dose-dependent (Table 1). Low doses of antigen administration}

favor the induction of active cellular regulation $(7,8)$, whereas higher doses favor the induction of clonal anergy (9) or deletion (10). Although important principles regarding oral tolerance were described in the 1970s and 1980s, most of these early studies of oral tolerance did not distinguish dose effects (11). To exploit oral tolerance in treating autoimmune or inflammatory diseases, it will be necessary to understand the various responses that are induced or suppressed during this process, beginning when antigen first encounters gut-associated lymphoid tissue (GALT). This well-developed immune network consists of lymphoid nodules (Peyer's patches), epithelial villi, intraepithelial lymphocytes, and other lymphocytes scattered throughout the lamina propria. Antigens may act directly at the level of the GALT or may exert their effects after absorption. Although dietary antigens are degraded by the time they reach the small intestine, studies in humans and rodents indicate that degradation is partial and that some intact antigen is absorbed, especially when large doses of antigen are fed $(12,13)$. In this regard, "oral tolerance" and "mucosal immunization" are part of one immunologic continuum, which must be explained with reference to antigenpresenting cell interactions with $\mathrm{T}$ cells

Table 1

Mechanisms of immune tolerance and their relationship to oral tolerance

$\begin{array}{lc}\text { Mechanism of tolerance } & \text { Dose administered } \\ \text { Deletion } & \text { High dose } \\ \text { Anergy } & \text { High dose } \\ \text { Receptor downregulation } & \text { High dose } \\ \text { Cellular regulation } & \\ \text { Th2 cells (IL-4) } & \text { Low dose } \\ \text { Th3 cells (TGF- } \beta \text { ) } & \text { Low dose } \\ \text { Tr1 cells (IL-10) } & \text { Low dose } \\ \text { CD2 } 25^{+} \text {CD4 } 4^{+} \text {cells } & \text { High/low dose }\end{array}$

The dose relationships may be modified by adjuvants, antigen structure, and dosing frequency. 
in the GALT. In addition to antigen dose, the nature of the antigen, the innate immune system, the genetic background and immunologic status of the host, and mucosal adjuvants influence the immunologic outcome following oral antigen administration. In the current issue of the JCI, Benson et al. offer a detailed explanation of a mechanism by which high-dose oral tolerance occurs (14). Working with transgenic mice whose $T$ cells carry a Tcell receptor (TCR) specific for myelin basic protein (MBP), these authors show that T-cell activation and receptor downmodulation occur after animals are exposed to high oral doses of MBP but that they precede deletion of the $T$ cells. Because the animals underwent thymectomy in adulthood, prior to feeding with MBP, Benson et al. (14) were able to follow the responses of circulating $\mathrm{T}$ cells, independent of any effects of new thymic emigrants. One day after exposure to the oral antigen MBP, they find that these T cells downregulate their TCR, which returns to the cell surface by 3 days after feeding. These cellular events dramatically affect the course of autoimmune responses: Animals that were injected with the antigen on day 1 after the feeding were protected from developing experimental autoimmune encephalomyelitis (EAE; an animal model for multiple sclerosis [MS]), whereas those immunized on day 3 were not. Similarly, T cells taken from animals on day 3 could be used to passively transfer EAE to naive host animals, but cells taken on day 1 could not. The transient decrease of MBP-reactive T cells on day 1 was followed by an intermediate state of anergy and, later, deletion, which occurred by 14 days after feeding. Furthermore, these cells expressed markers characteristic of activated T cells (CD69 accompanied by a decrease of CD62L), suggesting that they had not been triggered to become memory cells. Clearly, tolerance in the immediate aftermath of MBP feeding arose not from T-cell deletion or anergy, but from receptor downmodulation, which could be observed in the increased levels of intracellular TCRs.

\section{Qualitative changes in T cell responses}

Cytokines play an important role in tolerance mechanisms, especially those involving active cellular regulation. In addition, there may be selective induction of tolerance for cell-mediated versus antibody responses, and for Th1like, rather than Th2-like, responses. Benson et al. (14) did not report the effect of their feeding regimen on humoral immunity, but one presumes that antibody responses were suppressed. They did, however, carry out detailed analysis of cytokine responses following a single high-dose feeding. They found that on day 1 there was an increase in the number of cells expressing IL-2, IFN- $\gamma$, IL-5, and IL-4, followed by their subsequent decrease and apparent deletion by day 14 , a consequence of apoptosis. Cells secreting TGF- $\beta$, referred to as Th3 cells, have been implicated in the active suppression mechanism of oral tolerance (7); in the present report (14), these cells were distinctive in that they did not undergo deletion following oral exposure to MBP, consistent with findings in previous studies (10).

The studies of Benson et al. (14), demonstrating TCR downmodulation as an immediate but reversible effect, help to define the mechanisms of oral tolerance associated with high-dose feeding. This effect is associated with protection from disease and represents the first step toward anergy and deletion. The present studies also show that large doses of orally administered antigen act systemically and can suppress both Th1 and Th2 responses, and they highlight the unusual nature of the not yet completely understood Th3 class of immune cells. In addition, as more is learned about mechanisms of cellular regulation, the traditionally sharp distinction between anergic and regulatory cells is beginning to blur. Other investigators have reported that anergic cells can mediate active suppression $(15,16)$. Furthermore, an important class of regulatory cells, $\mathrm{CD}_{25}{ }^{+} \mathrm{CD} 4{ }^{+}$cells, are anergic and have suppressive properties that may act in a nonspecific fashion (17), perhaps in part because they express RNA for cytokines such as TGF- $\beta$ and IL-10 (18). We recently reported that CD $25^{+} \mathrm{CD} 4^{+}$regulatory cells can be induced following oral antigen administration in ovalbumin TCR transgenic mice (19), raising the possibility that these cells could participate in oral tolerance induction to MBP in the EAE model as well.

\section{Oral tolerance in clinical applications}

Tolerance following mucosal administration of antigens poses a number of questions of basic biologic importance and has also begun to take on major clinical importance as mucosal tolerance (both oral and nasal administration) has been applied to human diseases. Indeed, mucosal administration of antigens has been shown in animal models to ameliorate not only classic autoimmune processes, but also stroke (20), Alzheimer's disease (21), and, more recently, atherosclerosis (22). In each of these cases, understanding the mechanisms of action will be crucial for the successful application of these therapies to humans.

The work by Benson et al. (14) sheds important light on mechanisms of tolerance following oral antigen and on the qualitatively different effects of varying antigen doses. In particular, the present findings raise the possibility that Th2type responses in people might also be successfully suppressed by high doses of antigen, a finding reported by other investigators (23). Thus far, multiple doses of antigen have been used for treating human conditions, and it will be important to understand immunologic mechanisms to monitor dose effects properly. Other dosing regimens, such as continuous feeding to provide prolonged exposure of the gut to oral antigens, have also been tested in animals and have been shown to suppress Th2 type responses (24) and to be associated with increased TGF- $\beta$ (25). It will be of interest to know whether TCR downregulation and clonal deletion also occur under these conditions. In initial human trials in MS, an oral myelin preparation did not appear to block progression of MS, but this disappointing finding may be due to the use of an inadequate dose or to the type of antigen administered (6). Currently, a multicenter, 1300-patient, phase III clinical trial of an orally administered MBP analogue, glatiramer acetate, is in progress, based on positive effects observed in the animal model $(26,27)$. Two doses are being administered on a daily basis for a 14-month period, and it will be interesting to learn whether one dose is more efficacious. In addition, an NIH-sponsored multicenter diabetes prevention trial is in progress in which oral insulin is being given to children at risk for developing type 1 diabetes. 
The mucosal route is extremely attractive from a clinical standpoint, as it is easily administered to patients and accesses a major part of the immune system. It is not clear how much the immunologic effects of large doses of oral antigen differ from the effects of antigen given by the intravenous route, although the advantage for clinical application and safety is obvious. Whitacre and her colleagues have been pioneers both in the study of oral tolerance in the EAE model (28) and in establishing the high-dose mechanism of anergy (9). Their continued work further defines basic mechanisms associated with orally administered antigen, which should hasten the development of mucosally administered antigens for the treatment of human diseases.

1. Burnet, M. 1959. The clonal selection theory of acquired immunity. Vanderbilt University Press. Nashville, Tennessee, USA.

2. Avrameas, S. 1991. Natural autoantibodies: from 'horror autotoxicus' to 'gnothi seauton.' Immunol. Today. 5:154-159.

3. Zhang, J., et al. 1993. Increased frequency of IL-2 responsive T-cells specific for myelin basic protein and proteolipid protein in peripheral blood and cerebrospinal fluid of patients with multiple sclerosis. J. Exp. Med. 179:973-984.

4. Cohen, I., and Young, D.B. 1991. Autoimmunity, microbial immunity and the immunological homunculus. Immunol. Today. 12:105-110.

5. Matzinger, P. 1994. Tolerance, danger, and the extended family. Annu. Rev. Immunol. 12:991-1045.
6. Faria, A.M.C., and Weiner, H.L. 1999. Oral tolerance: mechanisms and therapeutic applications. Adv. Immunol. 73:153-264.

7. Chen, Y., Kuchroo, V.K., Inobe, J.-I., Hafler, D.A., and Weiner, H.L. 1994. Regulatory T-cell clones induced by oral tolerance: suppression of autoimmune encephalomyelitis. Science. 265:1237-1240.

8. Friedman, A., and Weiner, H. 1994. Induction of anergy or active suppression following oral tolerance is determined by antigen dosage. Proc. Natl. Acad. Sci. USA. 91:6688-6692.

9. Whitacre, C.C., Gienapp, I.E., Orosz, C.G., and Bitar, D. 1991. Oral tolerance in experimental autoimmune encephalomyelitis. III. Evidence for clonal anergy. J. Immunol. 147:2155-2163.

10. Chen, Y., et al. 1995. Peripheral deletion of antigen-reactive T-cells in oral tolerance. Nature. 376:177-180.

11. Mowat, A.M. 1987. The regulation of immune responses to dietary protein antigens. Immunol. Today. 8:93-98.

12. Bruce, M.G., and Ferguson, A. 1986. Oral tolerance to ovalbumin in mice: studies of chemically modified and 'biologically filtered' antigen. Immunology. 4:627-630.

13. Bruce, M.G., and Ferguson, A. 1986. The influence of intestinal processing on the immunogenicity and molecular size of absorbed, circulating ovalbumin in mice. Immunology. 2:295-300.

14. Benson, J.M., et al. 2000. T-cell activation and receptor downmodulation precede deletion induced by mucosally administered antigen. $J$. Clin. Invest. 106:1031-1038.

15. Taams, L.S., et al. 1998. Anergic T-cells actively suppress T-cell responses via the antigen-presenting cell. Eur. J. Immunol. 28:2902-2912.

16. Jordan, M.S., Riley, M.P., von Boehmer, H., and Caton, A.J. 2000. Anergy and suppression regulate CD4(+) T cell responses to a self peptide. Eur J. Immunol. 30:136-144.

17. Thornton, A.M., and Shevach, E.M. 2000. Suppressor effector function of CD4+CD25+ immunoregulatory $\mathrm{T}$ cells is antigen nonspecific J. Immunol. 1:183-90.

18. Asano, M., Toda, M., Sakaguchi, N., and Sak- aguchi, S. 1996. Autoimmune disease as a consequence of developmental abnormality of a $\mathrm{T}$ cell subpopulation. J. Exp. Med. 12:387-396.

19. Zhang, X., Liu, L., Oida, T., and Weiner, H.L. 2000. Oral tolerance induces regulatory CD25+ CD4+ $\mathrm{T}$ cells in ovalbumin TCR transgenic mice. FASEB J. 14:A1198. (Abstr.)

20. Becker, K.J., et al. 1997. Immunologic tolerance to myelin basic protein decreases stroke size after transient focal cerebral ischemia. Proc. Natl. Acad. Sci. USA. 20:10873-10878.

21. Weiner, H.L., et al. 2000. Nasal administration of amyloid-beta peptide decreases cerebral amyloid burden in mouse model of Alzheimer's disease. Ann. Neurol. 48:567-579.

22. Maron, R., et al. 2000. Mucosal administration of HSP 65 decreases atherosclerosis and inflammation in the aortic arch of LDL receptor deficient mice. FASEB J. 14:A1199. (Abstr.)

23. Garside, P., et al. 1995. T helper 2 cells are subject to high dose oral tolerance and are not essential for its induction. J. Immunol. 154:5649-5655.

24. Melamed, D., Fishman-Lovell, J., Uni, Z., Weiner, H.L., and Friedman, A. 1996. Peripheral tolerance of Th2 lymphocytes induced by continuous feeding of ovalbumin. Int. Immunol. 5:717-724.

25. Faria, A.M.C., Maron, R., Komagata, Y., and Weiner, H.L. 1999. Continuous feeding of low dose ovalbumin enhances TGF- $\beta$ production by spleen cells of BALB/c, C57BL/ 6 and OVA TcR transgenic mice. FASEB J. 13:A608. (Abstr.)

26. Teitelbaum, D., Arnon, R., and Sela, M. 1999. Immunomodulation of experimental autoim mune encephalomyelitis by oral administration of copolymer 1. Proc. Natl. Acad. Sci. USA. 7:3842-3847.

27. Weiner, H.L. 1999. Oral tolerance with Copolymer 1 for the treatment of multiple sclerosis Proc. Natl. Acad. Sci. USA. 7:3333-3335.

28. Bitar, D.M., and Whitacre, C.C. 1988. Suppression of experimental autoimmune encephalomyelitis by the oral administration of myelin basic protein. Cell. Immunol. 2:364-370. 